\title{
A Compton-Vetoed Germanium Detector with Increased Sensitivity at Low Energies
}

S. Friedrich, C. Bates, O. B. Drury, M. Burks, D. DiPrete

April 16, 2012

Methods and Applications of Radioanalytical Chemistry IX Kona, HI, United States

March 25, 2012 through March 30, 2012 
This document was prepared as an account of work sponsored by an agency of the United States government. Neither the United States government nor Lawrence Livermore National Security, LLC, nor any of their employees makes any warranty, expressed or implied, or assumes any legal liability or responsibility for the accuracy, completeness, or usefulness of any information, apparatus, product, or process disclosed, or represents that its use would not infringe privately owned rights. Reference herein to any specific commercial product, process, or service by trade name, trademark, manufacturer, or otherwise does not necessarily constitute or imply its endorsement, recommendation, or favoring by the United States government or Lawrence Livermore National Security, LLC. The views and opinions of authors expressed herein do not necessarily state or reflect those of the United States government or Lawrence Livermore National Security, LLC, and shall not be used for advertising or product endorsement purposes. 


\title{
A Compton-Vetoed Germanium Detector with Increased Sensitivity at Low Energies (MARC IX Conference, log number 414)
}

\author{
S. Friedrich \\ friedrich1@1lnl.gov \\ C. R. Bates \\ batesca@berkeley.edu \\ M. T. Burks \\ burks5@1lnl.gov \\ O. B. Drury \\ drury2@1lnl.gov
}

Lawrence Livermore National Laboratory, 7000 East Ave., Livermore, CA 94550

\author{
D. P. DiPrete \\ david.diprete@srnl.doe.gov \\ Savannah River National Laboratory, Aiken, SC 29802
}

Corresponding Author: Stephan Friedrich, LLNL, 7000 East Ave., L-550, Livermore, CA 94550, USA; Email: friedrich1@1lnl.gov, +1 (925) 423-1527 (phone), +1 (925) 424-5512 (fax) 


\title{
A Compton-Vetoed Germanium Detector with Increased Sensitivity at Low Energies
}

\author{
S. Friedrich ${ }^{1}$, C. R. Bates ${ }^{1,2}$, M. T. Burks ${ }^{1}$, O.B. Drury ${ }^{1}$, D. P. DiPrete ${ }^{3}$ \\ ${ }^{1}$ Lawrence Livermore National Laboratory, Livermore, CA 94550 \\ ${ }^{2}$ University of California, Berkeley, CA 94720 \\ ${ }^{3}$ Savannah River National Laboratory, Aiken, SC 29802
}

The difficulty to directly detect plutonium in spent nuclear fuel due to the high Compton background of the fission products motivates the design of a Gamma detector with improved sensitivity at low energies. We have built such a detector by operating a thin high-purity Ge detector with a large scintillator Compton veto directly behind it. The Ge detector is thin to absorb just the low-energy Pu radiation of interest while minimizing Compton scattering of highenergy radiation from the fission products. The subsequent scintillator is large so that forwardscattered photons from the Ge detector interact in it at least once to provide an anti-coincidence veto for the Ge detector. For highest sensitivity, additional material in the line-of-sight is minimized, the radioactive sample is kept thin, and its radiation is collimated. We will discuss the instrument design, and demonstrate the feasibility of the approach with a prototype that employs two large CsI scintillator vetoes. Initial spectra of a thin Cs-137 calibration source show a background suppression of a factor of $\sim 2.5$ at $\sim 100 \mathrm{keV}$, limited by an unexpectedly thick $4 \mathrm{~mm}$ dead layer in the Ge detector.

Key words: isotope analysis, germanium detectors, Compton veto, background suppression, spent fuel analysis, plutonium detection 


\section{INTRODUCTION}

Gamma spectroscopy with high-purity germanium detectors can be limited in sensitivity at low energies by the Compton background from high-energy radiation. This prevents, for example, the direct detection of plutonium in spent nuclear fuel due to the high-intensity Compton background from the fission products. This problem is of significant concern in nuclear safeguards, since it limits the accuracy of the Pu assay and thus the accounting for tons of fissile material worldwide [1].

The sensitivity at low energies can be greatly increased with a large Compton veto located behind the primary Ge detector, which selectively rejects low-energy background events in the Ge by anti-coincidence vetoing $[2,3]$. We have designed such a composite detector that is optimized for background suppression at low energies $(\leq 200 \mathrm{keV})$ where the characteristic actinide lines of interest lie. Here we discuss the design considerations for increased sensitivity at low energies, and demonstrate the feasibility of the approach with a prototype instrument that employs a dual CsI scintillator veto.

\section{INSTRUMENT DESIGN}

The instrument design exploits the idea that the low-energy Compton background in Ge detectors is caused by forward-scattering of high-energy gamma-rays. A scintillator anticoincidence veto should therefore be placed behind the primary Ge detector, rather than around it as is typical for general-purpose Compton shields $[4,5]$. The Ge detector itself should be just thick enough to absorb the low-energy $\mathrm{Pu}$ radiation of interest without providing unnecessary extra mass for Compton scattering. Additional material in the line of sight between the source and the detectors should be minimized to reduce scattering to low energies in material other than 
the Ge detector, which cannot be vetoed. This includes the windows of the cryostat, the support structure of the Ge crystal, the case of the scintillator and the radioactive source itself. Scattering can be further reduced when the collimator between the source and the Ge detector is conical so that its inside is not illuminated directly. Additional shielding can be placed around the source to suppress backscattering from the laboratory environment, as long as the shield is open in the back to avoid direct Compton scattering from the shield into the detector (Figure 1).

Geant4 Monte-Carlo simulations illustrate the background suppression that can be achieved with a $10 \mathrm{~cm}$ (diameter) $\times 14 \mathrm{~cm}$ (length) scintillator behind an $8 \mathrm{~mm}$ thick Ge detector, assuming idealized conditions of zero additional mass in the line-of-sight between them and the source (Figure 2). The high-energy background, which is caused by large-angle scattering that bypasses the veto, is largely unaffected. The background starts to decrease at energies below $\sim 350 \mathrm{keV}$, which corresponds to a scattering angle of $\sim 90$ degrees where the scattered radiation starts to pass through the veto. The low-energy background can be suppressed by several orders of magnitude, depending on the size and the absorption efficiency of the scintillator material. The suppression is largest for a scattering angle towards the corner of the cylindrical veto where the path through the scintillator is longest and the probability of interaction is therefore highest (figure 1, red arrow).

In this idealized case, the background suppression can be estimated analytically from the ratio of interacting to transmitted photons in the scintillator according to

$$
\frac{B_{\text {NoVeto }}}{B_{\text {Veto }}}=\frac{1-e^{-\mu(E) \rho L(E)}}{e^{-\mu(E) \rho L(E)}} .
$$

Here $\mu(E)$ and $\rho$ are absorption coefficient and density of the scintillator material, and $L(E)$ is the length of the scintillator in direction of the scattering angle for Compton photons of energy $E$. 
The analytical estimate according to equation (1) is shown in grey in figure 2 for the case of a $\mathrm{NaI}$ veto at a distance of 5 and $7 \mathrm{~mm}$ from the Ge detector.

For more realistic cases with a finite amount of intervening material between the source and the detectors, the background suppression is reduced by the fraction of photons that interact in the intervening material. This adds a term $\Sigma\left(1-e^{-\mu(E) \rho T}\right)$ to the denominator of equation (1), where $\mu_{i}(E), \rho_{i}$ and $T_{i}$ are the absorption coefficient, density and thickness of the intervening materials, and the summation extends over all materials in the line of sight.

The degree to which the idealized case can be implemented in practice depends on how much the amount of intervening material in the line-of-sight can be reduced. We have built a prototype instrument to examine this question experimentally (Figure 3). It uses an $8 \mathrm{~mm}$ thick planar Ge detector with a diameter of $35 \mathrm{~mm}$ that is read out with a custom-designed preamplifier at room temperature. The Ge crystal is held and heat sunk to the $1 \mathrm{~N}_{2}$ cold stage by three plastic clamps at its edges that are not illuminated directly by the source. Two $0.025 \mathrm{~mm} \mathrm{Al}$ windows in front and behind the Ge detector reduce IR heating to the detector cold stage, and the vacuum barrier in the line-of-sight consists of two $0.75 \mathrm{~mm} \mathrm{Al}$ windows with $40 \mathrm{~mm}$ diameter. The source is collimated onto the central part of the Ge detector by a $10 \mathrm{~cm}$ thick $10 \mathrm{~cm}$ diameter tungsten collimator (Hevimet ${ }^{\circledR}$ MT-185) that has a conical aperture with a $12.5 \mathrm{~mm}$ entrance and a $25 \mathrm{~mm}$ exit opening. Sources that are placed at least $10 \mathrm{~cm}$ away from the entrance opening within the cone of the collimator will therefore not illuminate its inside directly (cf. Figure 1). The primary veto consists of a $3.5^{\prime \prime} \times 6^{\prime \prime} \mathrm{CsI}$ scintillator in a $0.8 \mathrm{~mm} \mathrm{Al} \mathrm{housing.} \mathrm{It} \mathrm{is}$ surrounded by a secondary annular CsI veto with an outer diameter of 5.5". All detectors are biased and read out with standard commercial electronics. 


\section{RESULTS AND DISCUSSION}

We have tested the response of the instrument with a $5 \mu \mathrm{Ci}$ Cs- 137 calibration source supported between two clear plastic sheets. The source is so dilute to be in invisible to the naked eye and can be considered a point source with negligible in-source scattering. We have also measured the laboratory background radiation, which is not negligible in this setup, since the source is weak and held at a distance of $\sim 25 \mathrm{~cm}$ from the Ge detector to avoid illuminating the inside of the W collimator. Figure 4 shows the background-corrected Cs-137 gamma spectra without the vetoes (dark green), and with both CsI vetoes installed and active (light green). It also shows Geant4 Monte-Carlo simulations of our geometry for the two cases, redistributed with a FWHM of $2.5 \mathrm{keV}$ to account for the finite energy resolution of the Ge detector (dark and light grey). All spectra are normalized to $10^{5}$ counts in the photopeak at $662 \mathrm{keV}$ (which causes the noise in the simulations to be unrealistically low) to allow a comparison of the Compton background level.

As expected, the spectra show the broad background with the Compton edge at $470 \mathrm{eV}$ and a small backscatter peak at $\sim 190 \mathrm{eV}$. The background is suppressed preferentially at low energies caused by to forward-scattered Compton photons. The absence of W X-rays at $\sim 60 \mathrm{keV}$ shows that the collimator works as expected without contributing any X-rays to the background. Still, the two vetoes suppress the background in the region of interest around $100 \mathrm{keV}$ only by a factor of $\sim 2.5$, significantly less than the expected factor of $\sim 15$ for this geometry based on the Monte-Carlo simulations. This limitation turns out to be due to an unexpectedly large dead layer at the back side electrode of the Ge detector. The thickness of such a dead layer can easily be measured in our geometry by comparing spectra for front and back-side illumination. Figure 5 shows two spectra from an Am-241 / Eu-152 calibration source and the measured line ratio at 
low energies. The observed line ratios (squares) are explained well by the absorption of a $4 \mathrm{~mm}$ dead layer (grey line), implying that half of the $8 \mathrm{~mm}$ Ge detector does not contribute to any signal, while fully contributing to the Compton background that cannot be vetoed. Including a uniform $4 \mathrm{~mm}$ Ge dead layer in the Monte-Carlo simulations qualitatively accounts for much of the discrepancy with the data, although it quantitatively differs significantly in the fine structure that it predicts in the Compton background. This implies that the dead layer inside the Ge detector is either not uniform, or that there are additional yet unidentified scattering sources in our setup. In future work, the Ge detector will therefore be replaced by a crystal with an amorphous Ge electrode whose dead layer should be only a few $\mu \mathrm{m}$ thick. In addition, the CsI vetoes will be replaced by $\mathrm{BGO}$ vetoes to further improve the background suppression at low energies, and the liquid $\mathrm{N}_{2}$ can be replaced by a mechanical cooler for improved deployability. If a suppression factor above $\sim 20$ at $\sim 100 \mathrm{keV}$ can be achieved, it would enable the direct detection of $\mathrm{Pu}$ lines for realistic spent nuclear fuel compositions.

\section{ACKNOWLEDGEMENTS}

We gratefully acknowledge the support of the U.S. Department of Energy, Office of Nuclear Energy under grant FTLL11MP0206 (MPACT). This work was performed under the auspices of the U.S. Department of Energy by Lawrence Livermore National Laboratory under Contract DE-AC52-07NA27344. 


\section{REFERENCES}

[1] D. REILLY, N. ENSSLIN, H. SMITH JR., S. KREINER, "PASSIVE NON-DESTRUCTIVE ASSAY OF NUCLEAR MATERIALS", Office of Nuclear Regulatory Research (NUREG/CR-5550), Washington, DC (1991)

[2] R. A. DEWBERRY, "A FORWARD-ANGLE $0.9 \pi$ ANTICOMPTON SPECTROMETER FOR ASSAY OF U-235 WITH FISSION-PRODUCTS CONTRIBUTIONS", Radioactivity and Radiochemistry 7, (1996) 27

[3] S. FRIEDRICH, "COMPOSITE HIGH-RESOLUTION GAMMA SPECTROMETER WITH INCREASED SENSITIVITY AT LOW ENERGIES", Patent application pending

[4] P. J. NOLAN, D. W. GIFFORD, P. J. TWIN, "THE PERFORMANCE OF A BISMUTH GERMANATE ESCAPE SUPPRESSED SPECTROMETER", Nuclear Instruments and Methods A236 (1985) 95

[5] S. G. PRUSSIN, M. D. PERRY, E. PEKRUL BICKNESE, J.F. LAMB, "A SMALL COMPTON SUPPRESSION SHIELD FOR Si(Li) DETECTORS", Nuclear Instruments and Methods A242 (1986) 410 


\section{LIST OF FIGURES}

Figure 1: Schematic cross section of the instrument

Figure 2: Monte-Carlo simulations of the Compton suppression that can be attained with different $10 \mathrm{~cm}$ (diameter) $\times 14 \mathrm{~cm}$ (length) scintillators under idealized conditions The grey lines are analytical approximations to the Compton suppression for a $\mathrm{NaI}$ scintillator veto 5 and $7 \mathrm{~mm}$ behind the Ge detector.

Figure 3: Picture of the prototype with the conical W collimator, the thin Ge detector, and the dual CsI veto directly behind it.

Figure 4: Background-corrected spectra from a Cs-137 source (green), compared to Geant4 Monte Carlo simulations without (grey), both with and without the two CsI vetoes.

Figure 5: Eu-152 / Am-241 spectra for front (dark) and back side (light green) illumination of the Ge detector. The observed line ratios (squares) correspond to a $4 \mathrm{~mm}$ dead layer on the back side electrode of the Ge detector (black). 
FIGURE 1

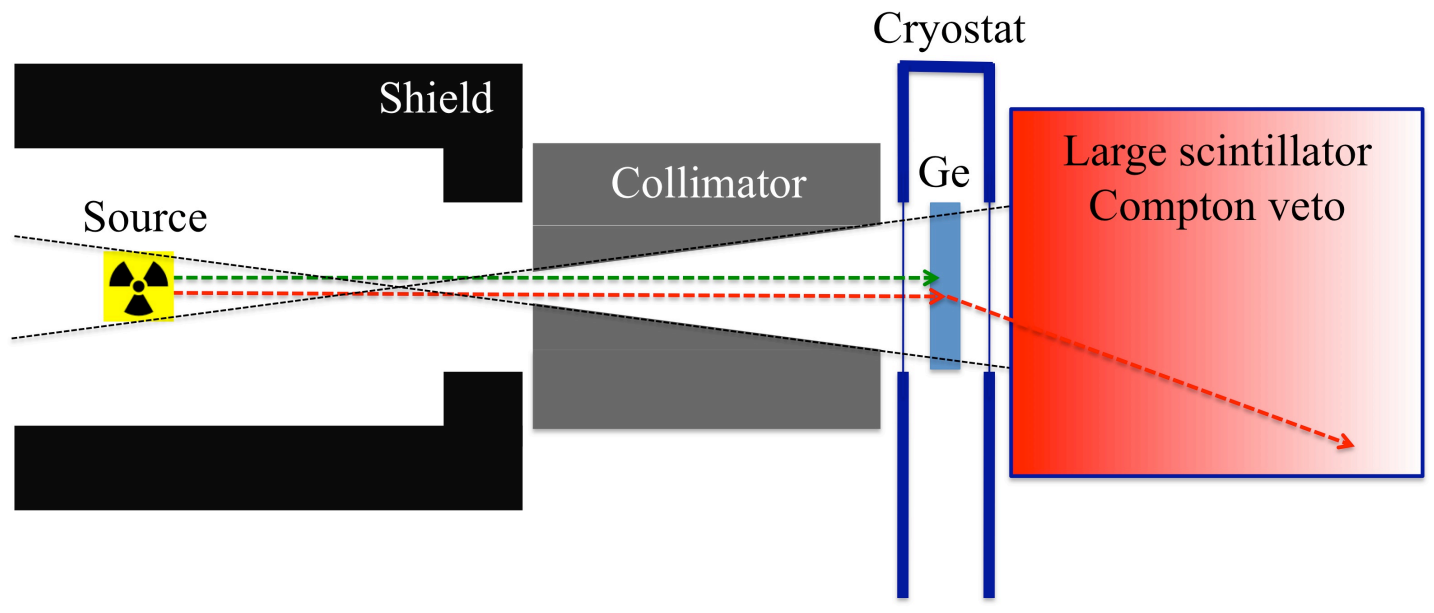


FIGURE 2

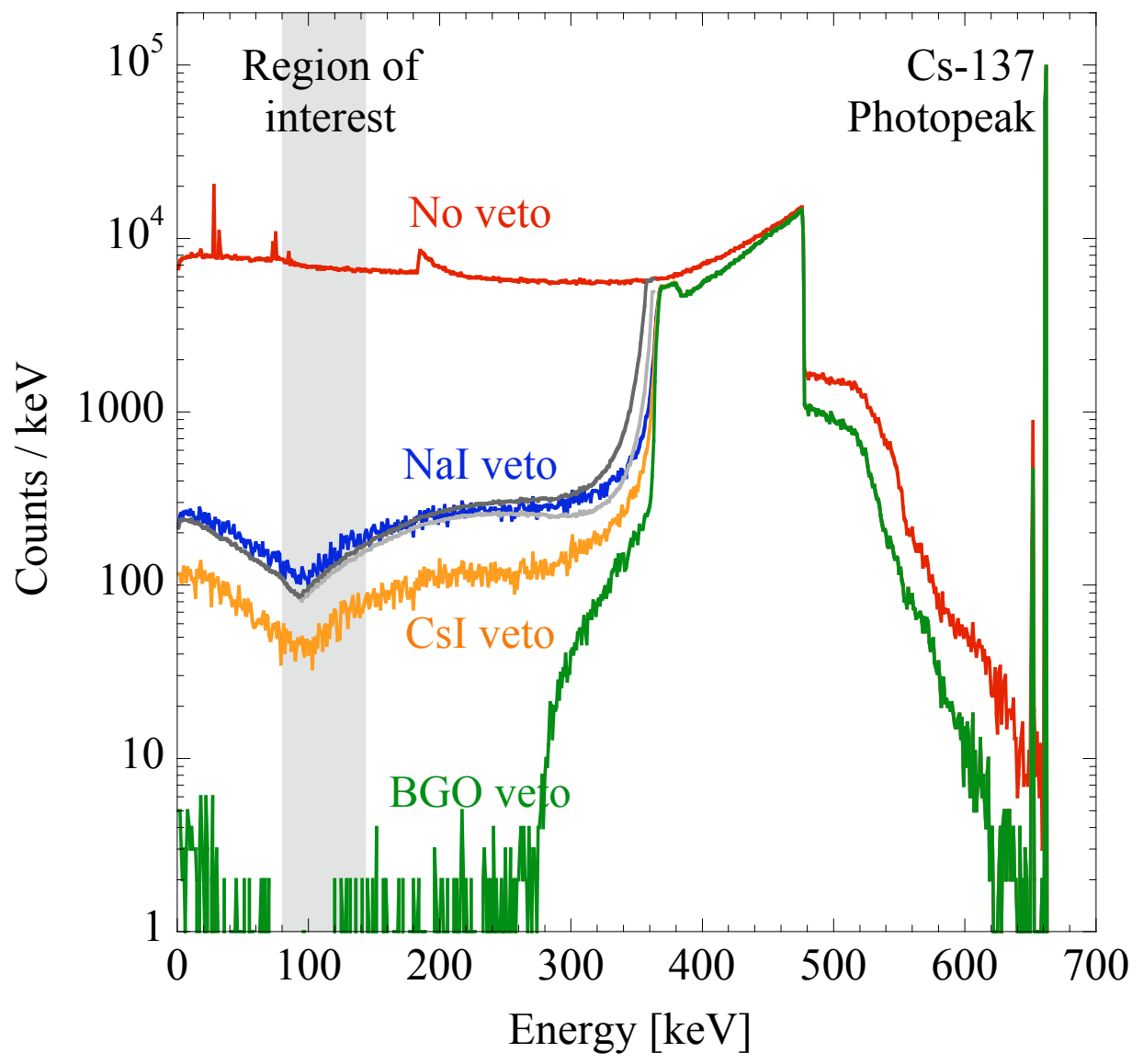


FIGURE 3

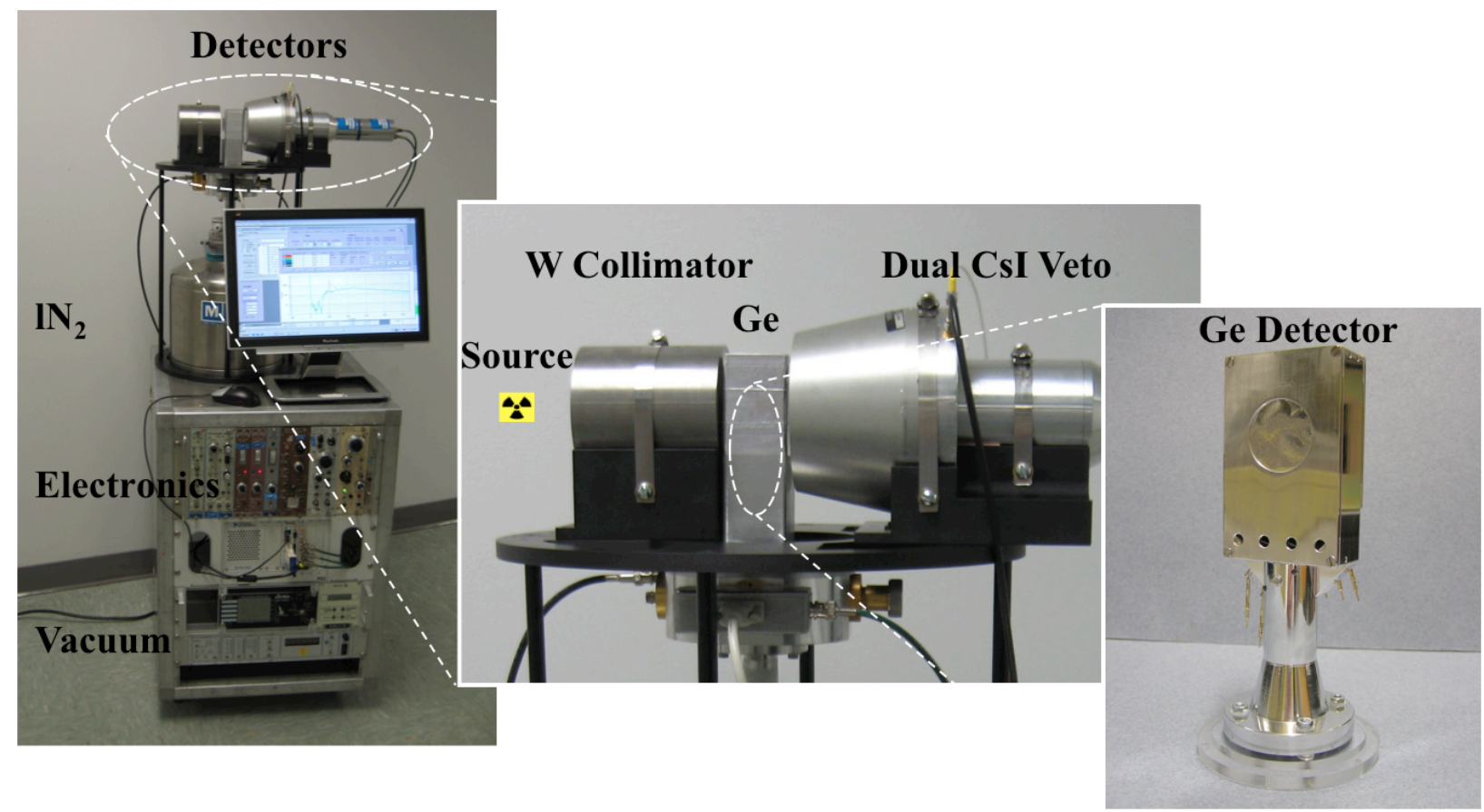


FIGURE 4

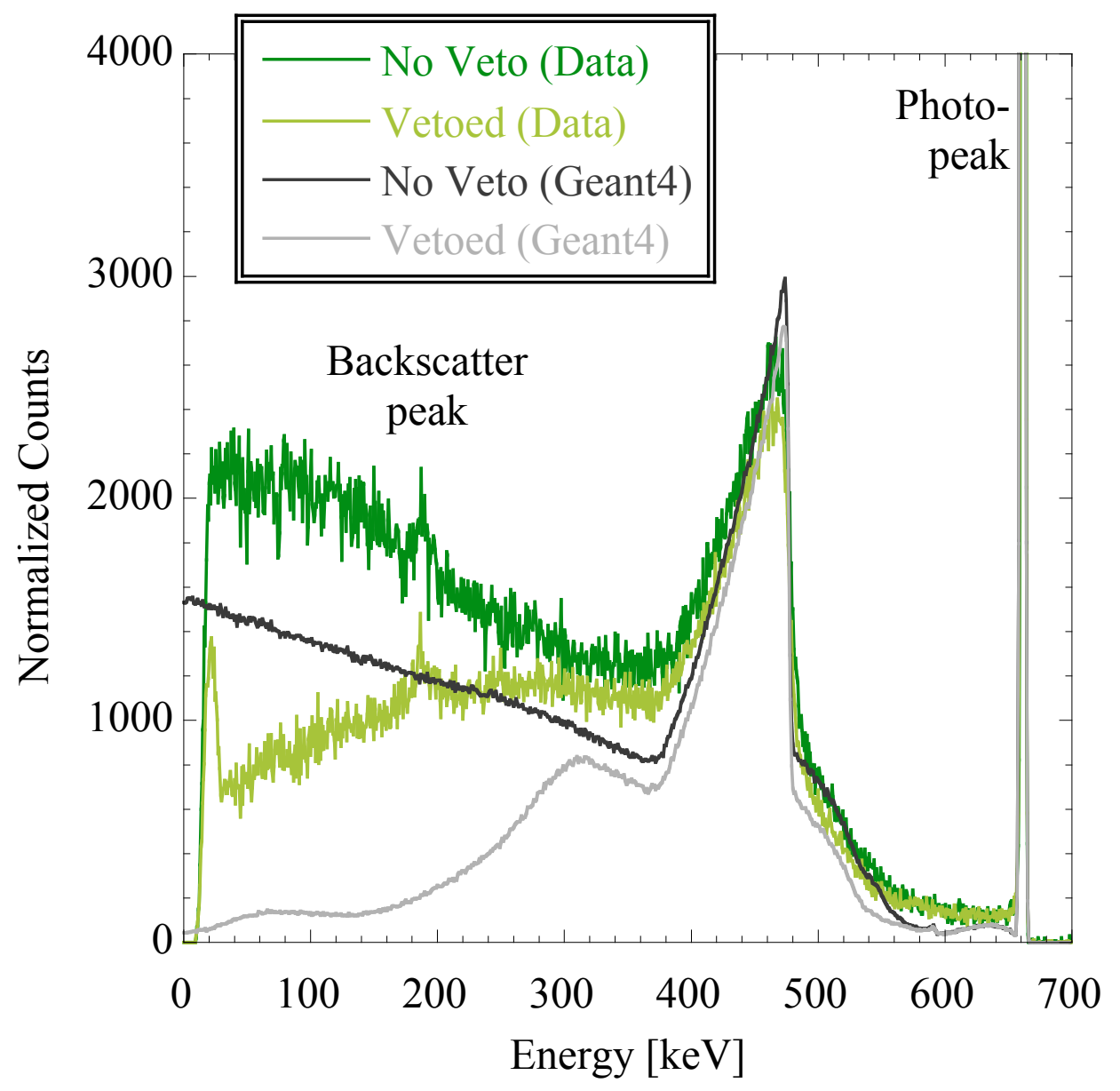


FIGURE 5

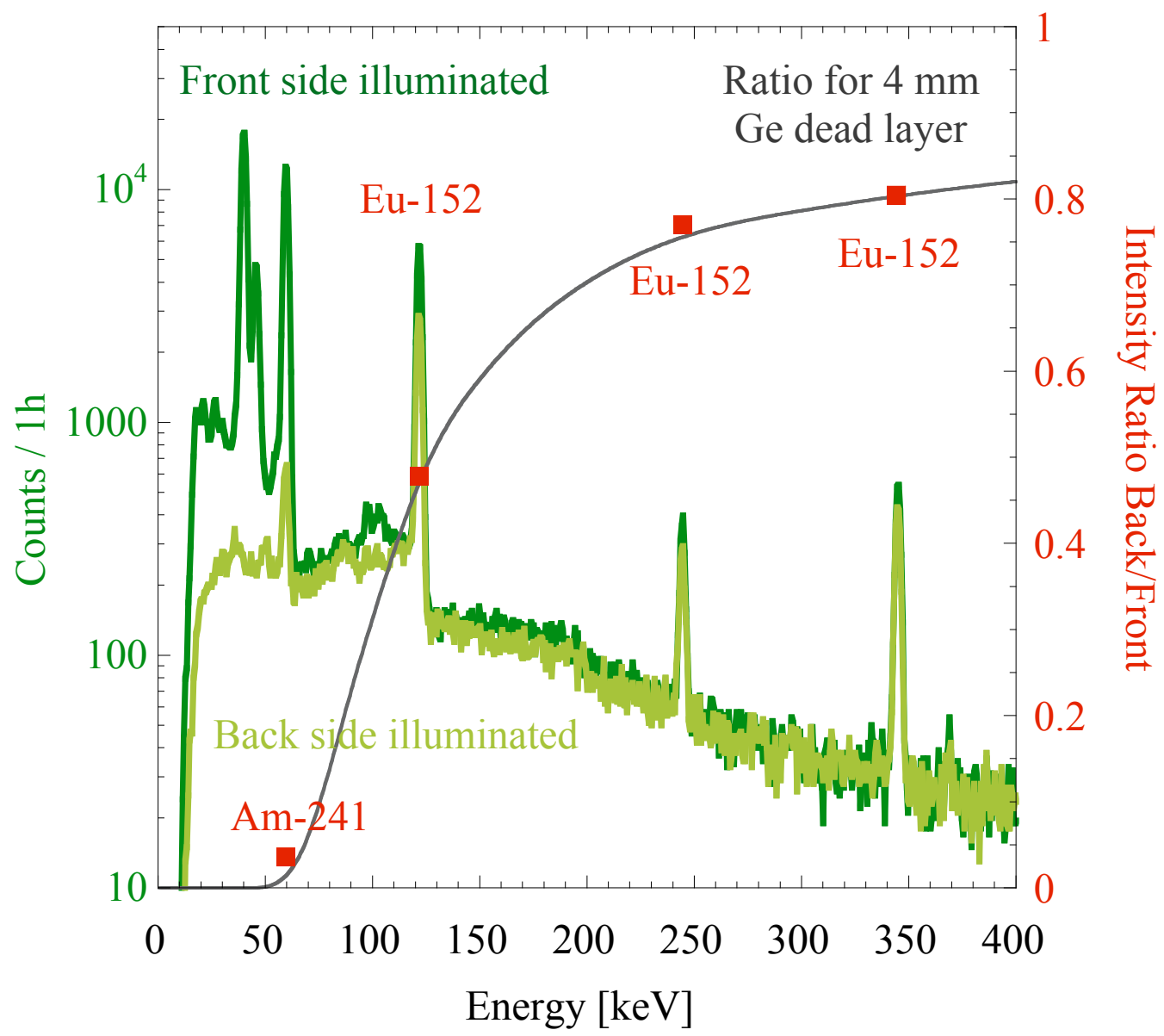

